\title{
Severe hemodynamic deterioration caused by cardiac herniation during endoscopic thoracic sympathicotomy in a patient with previously undiagnosed congenital pericardial defect
}

\author{
Joong-Ho Park, Eun-Ju Kim, Jong-Seouk Ban, Ji-Hyang Lee, and Ji-Hyun An \\ Department of Anesthesiology and Pain Medicine, Daegu Fatima Hospital, Daegu, Korea
}

Congenital pericardial defect is a rare but potentially fatal disorder as it can cause severe hemodynamic compromise. Its asymptomatic nature and lack of diagnostic roles in chest radiography or electrocardiography (ECG) make diagnosis difficult [1]. We report a case of a patient with previously undiagnosed pericardial defect, who developed ST elevation and severe hemodynamic deterioration during endoscopic thoracic sympathicotomy.

A 24-year-old male $(172.2 \mathrm{~cm}, 74.8 \mathrm{~kg})$ with a history of being a healthy hepatitis B carrier and having a congenital fusion of the cervical vertebrae was scheduled for repair of hyperhidrosis by endoscopic thoracic sympathicotomy. He had no previous cardiopulmonary symptoms, but preoperative ECG showed left posterior fascicular block. Chest radiography revealed an enlarged left atrial appendage (Fig. 1).

For premedication, glycopyrrolate $0.2 \mathrm{mg}$, midazolam 2.0 $\mathrm{mg}$, and famotidine $20 \mathrm{mg}$ were administered. Preoperative vital signs were blood pressure (BP) $110 / 70 \mathrm{mmHg}$, heart rate (HR) 64 beats per minute (bpm), and oxygen saturation $\left(\mathrm{SaO}_{2}\right)$ 98-99\%. General anesthesia was induced with propofol $120 \mathrm{mg}$ and rocuronium $50 \mathrm{mg}$. Intubation was performed using a 7.5 ID endotracheal tube. The operation began and the patient was placed in a reverse Trendelenburg position with the left side tilted upwards. To obtain a wider surgical field, carbon dioxide $\left(\mathrm{CO}_{2}\right)$ was insufflated into the thoracic cavity with a maximum pressure of $10 \mathrm{mmHg}$ at a flow rate of $1 \mathrm{~L} / \mathrm{min}$. Adhesiolysis was required since adhesions between the left lung and the medias- tinum hindered visualization of the sympathetic ganglion. The operation was uneventful until the left lung was pulled from the heart toward the chest wall during adhesiolysis, and the dissected cardiac border was protruding out of the mediastinum. Almost immediately, the patient's ECG developed ST elevation, and the BP abruptly dropped to $62 / 32 \mathrm{mmHg}$. The operation

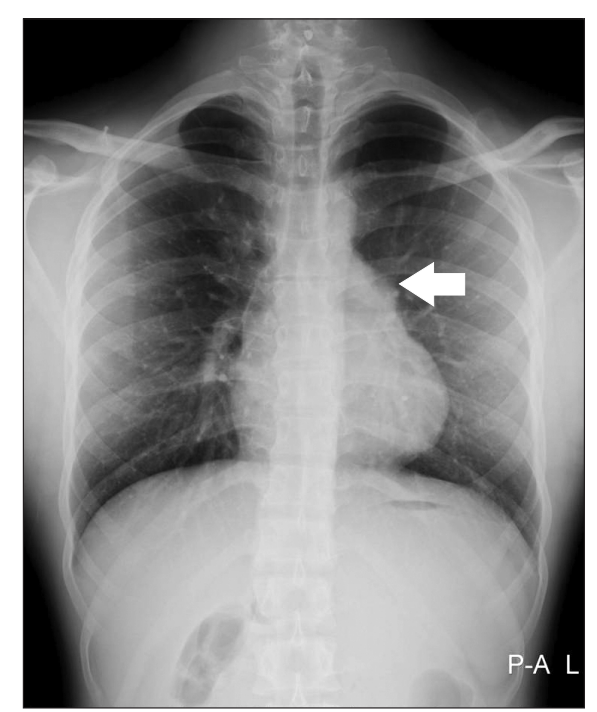

Fig. 1. Preoperative chest radiography shows enlarged left atrial appendage (arrow).

Corresponding author: Eun-Ju Kim, M.D., Department of Anesthesiology and Pain Medicine, Daegu Fatima Hospital, 99, Ayang-ro, Dong-gu, Daegu 701-724, Korea. Tel: 82-53-940-7436, Fax: 82-53-954-7417, E-mail: kej1127@fatima.or.kr

(c) This is an open-access article distributed under the terms of the Creative Commons Attribution Non-Commercial License (http:// creativecommons.org/licenses/by-nc/3.0/), which permits unrestricted non-commercial use, distribution, and reproduction in any medium, provided the original work is properly cited. 
was stopped and the patient was returned to the neutral supine position. Manual ventilation with a maximum pressure of 20 $\mathrm{mmHg}$ was performed. About a minute later, vital signs recovered, with BP 103/52 mmHg, HR $81 \mathrm{bpm}$, and $\mathrm{SaO}_{2}$ 98\%. The ECG returned to normal sinus rhythm. After discussion with the surgeon regarding the patient's condition, it was decided that the operation continue with the patient in the neutral supine position. Closer inspection of the cardiac border revealed complete absence of the left pericardium, with the origin of the left anterior descending artery abutting the mediastinum. After the operation of the left side was complete, the patient was tilted to the opposite position for the operation of the right side. No pericardial defect could be visually confirmed on this side, as massive amounts of adhesion were observed between the cardiac border and the lung. It was decided that the remainder of the operation continue without adhesiolysis on the right side, due to the risk of adverse events. The operation was completed without any further adverse events. The patient was taken to the postanesthesia care unit (PACU). One hour of observation in the PACU showed that the vital signs remained within the normal range. The patient was transferred to the general ward. He was discharged three days later without further complications.

In this case, the most likely cause of the adverse events appears to be the occurrence of cardiac herniation. The mechanisms and symptoms of cardiac herniation caused by pericardial defect vary depending on whether the defect is right-sided or left-sided [2]. In a right-sided herniation, dextrorotation of the heart may cause torsion of the atrio-caval junction, compression of the vena cava, and ventricular outflow obstruction, leading to severe decreases in cardiac output. In a left-sided herniation, pressure may be applied on the myocardium and coronary arteries by the edges of the pericardial defect, causing fatal ventricular outflow obstruction, arrhythmia, and myocardial infarction [2]. Normally, traction of the lung during adhesiolysis only pulls the pericardium, not the heart itself. However, in this case, absence of the pericardium may have caused the heart to slip out of the mediastinum during the traction of the left lung toward the chest wall. Obstruction of the ventricular outflow caused by cardiac herniation appears to have resulted in sudden decreases in BP. ST elevation was found in lead II, suggesting that either the posterior left ventricular artery of the right coronary artery or the left circumflex artery were the coronary arteries compressed. Recovery of the neutral position and manual insufflation of the lung appear to have placed the herniated heart back into the mediastinum.

Another possibility is that the insufflation of $\mathrm{CO}_{2}$ into the thoracic cavity may have created an atmosphere similar to a tension pneumothorax. Wolfer et al. [3] have documented that insufflation of $\mathrm{CO}_{2}$ under low pressure $(<10 \mathrm{mmHg})$ and low flow can be used safely. Thus in our patient, intrathoracic pressure was maintained at a maximum of $10 \mathrm{mmHg}$ through the use of low flow $\mathrm{CO}_{2}$ at a rate of $1 \mathrm{~L} / \mathrm{min}$. ECG changes were observed about 10 minutes after gas insufflation, which suggests that this case is different from the situations in which symptoms appear immediately after gas insufflation, and resolve after the release of the insufflated gas $[4,5]$. In this case, vital signs were recovered by returning the patient to the neutral supine position and performing manual insufflation of the lung, without any reduction in intrathoracic pressure. This also suggests that the symptoms were probably caused by the cardiac herniation, rather than reflecting cardiovascular collapse induced by gas insufflation.

It is difficult to diagnose pericardial defect preoperatively, but measures can be taken to reduce potential complications. Before undergoing surgical intervention and gas insufflation into the intrathoracic cavity, thorough evaluation employing echocardiography, chest computed tomography, and magnetic resonance imaging may be helpful. Careful monitoring throughout the course of the operation, particularly during events such as position changes and surgical manipulation, is also necessary.

Unlike other reports of cardiovascular collapse during thoracoscopic procedures [4,5], it appears that the absence of the pericardium resulting in cardiac herniation led to hemodynamic deterioration accompanied by ECG changes in our patient. We report this case as this phenomenon is considered rare but should not be overlooked.

\section{References}

1. Barçin C, Olcay A, Kocaoğlu M, Ataç K, Kurşaklioğlu H. Asymptomatic congenital pericardial defect: an aspect of diagnostic modalities and treatment. Anadolu Kardiyol Derg 2006; 6: 387-9.

2. Gadhinglajkar S, Siddappa S, Sreedhar R, Madathipat U. Cardiac herniation following completion pneumonectomy for bronchiectasis. Ann Card Anaesth 2010; 13: 249-52.

3. Wolfer RS, Krasna MJ, Hasnain JU, McLaughlin JS. Hemodynamic effects of carbon dioxide insufflation during thoracoscopy. Ann Thorac Surg 1994; 58: 404-7.

4. Harris RJ, Benveniste G, Pfitzner J. Cardiovascular collapse caused by carbon dioxide insufflation during one-lung anaesthesia for thoracoscopic dorsal sympathectomy. Anaesth Intensive Care 2002; 30: 86-9.

5. Grichnik KP, Dentz M, Lubarsky DA. Hemodynamic collapse during thoracoscopy. J Cardiothorac Vasc Anesth 1993; 7: 588-9. 